\title{
Novel combined approach in the management of non-healing solitary rectal ulcer syndrome - laparoscopic resection rectopexy and transanal endoscopic microsurgery
}

\author{
Petr Ihnat ${ }^{1,2}$, Lubomir Martinek ${ }^{3}$, Petr Vavra ${ }^{1,2}$, Pavel Zonca ${ }^{1,2}$ \\ ${ }^{1}$ Department of Surgery, University Hospital Ostrava, Ostrava, Czech Republic \\ 2Department of Surgical Studies, Faculty of Medicine, University of Ostrava, Ostrava, Czech Republic \\ ${ }^{3}$ Department of Surgery, $2^{\text {nd }}$ Faculty of Medicine, Charles University in Prague and Motol University Hospital, Prague, Czech Republic
}

Videosurgery Miniinv 2015; 10 (2): 295-298

DOI: $10.5114 /$ wiitm.2015.52060

\begin{abstract}
Solitary rectal ulcer syndrome (SRUS) is an uncommon chronic disorder with a wide range of endoscopic findings, clinical presentations and characteristic histopathological features. There is no clear consensus regarding SRUS management, because of its poorly understood pathogenesis and frequent association with various pelvic floor disorders. Laparoscopic resection rectopexy and transanal endoscopic microsurgery (TEM) were used for the treatment of non-healing SRUS. The present paper reports a case of non-healing SRUS due to obstructive defecation syndrome based on combined pelvic floor disorders (rectocele, enterocele, internal rectal prolapse and dolichosigma) successfully managed by a novel combined mini-invasive approach which has never been previously reported in the literature (laparoscopic resection rectopexy and TEM). The new minimally invasive concept seems to be safe and feasible - laparoscopic resection rectopexy results in effective correction of the obstructive defecation syndrome, while TEM allows comfortable access for radical resection of a rectal ulcer.
\end{abstract}

Keywords: solitary rectal ulcer, resection rectopexy, transanal endoscopic microsurgery.

\section{Introduction}

Solitary rectal ulcer syndrome (SRUS) is a rare chronic disorder characterized by a combination of symptoms, endoscopic findings and histological abnormalities [1-3]. The most common symptoms are constipation, rectal bleeding, mucous discharge, pain, and feeling of incomplete defecation. The SRUS is associated with a wide range of endoscopic findings such as erythematous patches, solitary or multiple ulcers, or polypoid growths [2,3]. That is why SRUS is also known as the "three-lie disease" (not always solitary, not always ulcerated, and not always located in the rectum) [4]. Characteristic histo- pathological features are fibromuscular obliteration of the lamina propria, hypertrophied muscularis mucosa and chronic inflammatory cell infiltration $[1,5]$.

The SRUS is difficult to treat because of its poorly understood pathogenesis and frequent association with various pelvic floor disorders. Optimal management is ambiguous, with no clear consensus. Several treatment options are available ranging from conservative management, biofeedback to a variety of surgical procedures (rectopexy, rectal excision with coloanal anastomosis, excision of the ulcer, Delorme's operation, defunctioning colostomy etc.). The widely accepted indication for surgery is conservative treatment failure $[1,3,5]$. At the present, the most pop- 
ular surgical techniques in SRUS management are laparoscopic rectopexy and stapled transanal rectal resection (STARR) $[1,3,6]$. Non-healing rectal ulcer presents a problematic issue because of possible invasive cancer development as a consequence of long-term chronic inflammation influence. If rectopexy fails to heal SRUS, STARR was advocated to be the suitable treatment option. However, STARR performance can be very difficult, especially if the ulcer is located far away from the anal verge.

Herein, we report a novel concept in the management of non-healing SRUS. Effective combination of mini-invasive techniques, laparoscopic resection rectopexy and transanal endoscopic microsurgery (TEM) has not been previously described in the literature. In the present case report we describe the operative technique, report the postoperative course of the patient, and discuss treatment options and patient's management rationale.

\section{Case report}

A 46-year-old woman presented with a 9-year history of severe chronic constipation based on an anorectal outlet obstruction. There was no history of mucous discharge or bleeding per rectum. Seven years ago, she underwent complete examination at a specialized coloproctology unit of another university hospital, which revealed solitary rectal ulcer associated with pelvic floor disorders - rectocele, enterocele and internal rectal prolapse. At that time, she had refused to undergo treatment because of her personal and career reasons. After 7 years of bearing impaired quality of life as a consequence of severe obstructed defecation syndrome, she changed her mind and came to our institution willing to undergo treatment.

Symptoms of constipation reached a score of 24 according to the Wexner Constipation Score. Colonoscopy revealed dolichosigma and ulcerated lesion $(3 \times 3 \mathrm{~cm})$ on the anterior wall of the rectum $7 \mathrm{~cm}$ from the anal verge. Multiple biopsies taken from the ulcer confirmed the diagnosis of SRUS by chronic inflammation and fibromuscular obliteration of the lamina propria findings. Colonic transit study showed obstructed defecation syndrome with normal colon transit time. Endoanal ultrasound, defecography and anal manometry confirmed presence of obstructive defecation syndrome based on rectocele, enterocele and internal rectal prolapse. Based on the prolonged history of known SRUS as- sociated with combined pelvic floor disorders, laparoscopic resection rectopexy was indicated as the first choice treatment option.

In brief, during laparoscopic resection rectopexy, the rectum was mobilized down to the pelvic floor using ultrasonic dissection with only partial division of the lateral ligaments and careful preservation of the ureters and autonomic nerves. The rectum was transected by an endostapler at the level of the promontorium, and a specimen was removed through an enlarged trocar incision in the left lower abdominal quadrant. The anastomosis was performed via standard double-stapler technique with anastomosis in the upper third of the rectum (preoperative rectoscopy confirmed colorectal anastomosis $14 \mathrm{~cm}$ from the anal verge, approximately $3 \mathrm{~cm}$ orally from the solitary rectal ulcer). Laparoscopic suture rectopexy was then performed - left and right lateral walls of the mobilized rectum (below the anastomosis) were fixed to the lateral peritoneal edge using running sutures. Postoperative recovery was uneventful; the patient was discharged 5 days after surgery.

Follow-up was done in the outpatient clinic at 1 , 3, 6 and 12 months. It revealed an excellent effect of resection rectopexy on obstipation symptoms - the Wexner constipation score decreased to 10 ( 1 month after surgery) and later to 6 (6 months after surgery). Defecography confirmed reparation of rectocele, enterocele and internal rectal prolapse. Despite a successful effect of laparoscopic resection rectopexy on obstructive defecation symptoms, repeated endoscopies showed a persistent solitary rectal ulcer with no tendency to heal even 1 year after the surgery.

Non-healing SRUS was the reason for secondary surgery indication 1 year after rectopexy. Transanal excision of rectal ulcer and STARR technique were rejected because of the great distance of the rectal ulcer from the anal verge $(7 \mathrm{~cm})$. The TEM technique was employed in order to achieve safe excision of the solitary rectal ulcer. In the jackknife position, full thickness excision of the rectal wall with ulcer and a 1-cm safety margin was performed by TEM, using ultrasonic dissection for safe haemostasis. The defect in the rectal wall was closed using absorbable running suture ended with silver clips. Postoperative recovery was uneventful; the patient was discharged 2 days after surgery. Histopathology examination confirmed diagnosis of SRUS with no signs of invasive carcinoma. 


\section{Discussion}

The SRUS is an interesting but poorly understood disorder which was first described by Madigan and Morson more than 40 years ago [7]. Endoscopic and clinical presentations may be confusing. The SRUS can present with diverse endoscopic findings, which is why it is necessary for clinicians to keep in mind the possibility of SRUS to reach the correct conclusion. Diagnosis is definitively confirmed by characteristic histopathology findings.

In the literature, there are conflicting opinions regarding its etiology and management. The pathogenesis of SRUS is not fully understood, and multiple factors may be involved. Patients often demonstrate abnormalities of the anal and pelvic floor musculature during defecation. That is why it seems that SRUS might be a secondary condition to functional evacuatory disorders rather than an independent entity [3]. The most accepted theories of SRUS development are related to repeated mucosal trauma and local ischemia of the rectal wall due to excessive prolonged straining at stool causing internal rectal prolapse $[1,5,8]$. Rectal prolapse, which is the most common underlying pathogenetic mechanism in SRUS, causes mucosal ischemia through venous congestion, retraction of submucosal vessels and fibroblasts replacing blood vessels. Local ischemia leads to mucosal edema with consequent ulceration $[1,8]$. Increased anal pressure and paradoxical puborectalis contraction during straining in patients with SRUS was found by Morio et al. [9].

There is very little definitive evidence regarding management of SRUS, with no clear consensus. Treatment options include behavioral modification, topical treatment, biofeedback and surgery. The SRUS management mostly depends on the severity of symptoms and whether there are associated pelvic floor disorders (rectal prolapse, rectocele, enterocele) $[1,5]$.

Surgery is reserved for patients not responsive to conservative treatment and biofeedback. Surgical approaches aimed to resolve SRUS can be divided into transabdominal and perineal procedures. Perineal procedures (such as local excision or STARR) have lower morbidity but higher recurrence rates with worse functional results in comparison with transabdominal procedures $[3,5,10,11]$. Because of greater understanding that rectal prolapse (internal or external) is the most important underlying mechanism of SRUS pathogenesis, the focus of surgical management has been shifted to rectopexy in the last decade. Stopping rectal prolapse may lead to rapid healing of the SRUS $[1,5]$.

In our case, laparoscopic resection rectopexy was the first-line treatment option due to the following reasons. The patient had symptoms of severe obstipation (Wexner constipation score 24) with verified obstructive defecation syndrome due to combined pelvic floor disorders (rectocele, enterocele, internal rectal prolapse). A perineal approach was excluded because of the presence of enterocele and dolichosigma. Regarding various types of laparoscopic rectopexy (such as suture rectopexy, mesh rectopexy or resection rectopexy) for patients with SRUS and combined pelvic floor disorders, resection rectopexy seems to be the most suitable technique, because it is reasonable to suppose that non-resection rectopexy (suture or mesh rectopexy) would probably lead to significant deterioration of symptoms of severe obstipation [11, 12].

Laparoscopic resection rectopexy aims to treat rectal prolapse and thus to promote rectal ulcer healing with simultaneous resolution of symptoms without inducing postoperative chronic obstipation. It has been successful, with a substantial proportion of patients having a marked reduction in symptoms and signs of ulcer healing. Especially Laubert and his colleagues reported excellent results of resection rectopexy in a single centre study on an impressive group of 264 patients with rectal prolapse [12].

In the management of patients with SRUS, successfulness of the treatment modality can be assessed by symptoms' resolution, endoscopic evidence of complete ulcer healing and improved quality of life. In our case, there was a substantial improvement in obstructive defecation symptoms and patients' quality of life, but there were no endoscopic signs of rectal ulcer healing. We suppose that the main reason for the non-healing character of solitary rectal ulcer in our patient was a very long history of rectal ulcer presence (the patient did not wish to undergo surgical treatment for more than 7 years). Despite elimination of the leading pathogenesis mechanism (rectal prolapse) via laparoscopic resection rectopexy, prolonged presence of chronic inflammation led to irreversible changes in the rectal wall which thwarted ulcer healing.

Paying attention to the proper management of non-healing rectal ulcer is very important because of potential invasive carcinoma development from 
the long-term perspective. Tsuchida et al. pointed out this possibility by describing the first case of SRUS associated with dysplasia and well-differentiated invasive adenocarcinoma [13]. Threat of invasive carcinoma development was the principal reason for the secondary surgery indication in our case. Transanal local excision of the non-healing rectal ulcer was not feasible because of the long distance between the ulcer and the anal verge (the aboral margin of the ulcer was within $7 \mathrm{~cm}$ and the oral margin at $10 \mathrm{~cm}$ from the anal verge). For the same reason, safe performance of STARR technique with clear resection margins was also very questionable. The TEM was therefore employed as the optimal surgical technique, allowing excellent access to rectal lesions located far away from the anal verge [14].

The TEM provides a safe and effective way of full thickness excision of the rectal wall with a $1-\mathrm{cm}$ margin. The technique facilitates the surgical approach to rectal lesions and allows rectal resection with a clear and detailed view $[14,15]$. Excellent haemostasis can be achieved efficiently using ultrasonic dissection; the rectal wall defect is closed safely and precisely. Because of these benefits, we believe that management of non-healing SRUS (if laparoscopic rectopexy fails to cure rectal ulcer) should be done by means of TEM.

\section{Conclusions}

The SRUS is a chronic benign disorder of the rectum. There is no clear consensus regarding SRUS management, because of its poorly understood pathogenesis and frequent association with various pelvic floor disorders. Herein, we present a novel combined minimally invasive approach for the management of non-healing SRUS, which has not been previously reported: laparoscopic resection rectopexy and TEM. The new concept seems to be safe, feasible and effective - laparoscopic resection rectopexy results in effective correction of obstructive defecation syndrome, while TEM allows comfortable access for radical resection of a rectal ulcer.

\section{Conflict of interest}

The authors declare no conflict of interest.

\section{References}

1. Zhu QC, Shen RR, Quin HL, Wang Y. Solitary rectal ulcer syndrome: clinical features, pathophysiology, diagnosis and treatment strategies. World J Gastroenterol 2014; 20: 738-44.
2. Abid S, Khawaja A, Bhimani SA, et al. The clinical, endoscopic and histological spectrum of the solitary rectal ulcer syndrome: a single-center experience of 116 cases. BMC Gastroenterol 2012; 12: 72.

3. Choi HJ, Shin EJ, Hwang YH, et al. Clinical presentation and surgical outcome in patients with solitary rectal ulcer syndrome. Surg Innov 2005; 12: 307-13.

4. Pérez LC, Vicente VM, Verge CR, et al. "The three-lies disease": solitary rectal ulcer syndrome. Rev Esp Enferm Dig 2007; 99: 663-6.

5. Evans C, Ong E, Jones OM, et al. Laparoscopic ventral rectopexy is effective for solitary rectal ulcer syndrome when associated with rectal prolapse. Colorectal Dis 2014; 16: 112-6.

6. Torres C, Khaikin M, Bracho J, et al. Solitary rectal ulcer syndrome: clinical findings, surgical treatment, and outcomes. Int J Colorectal Dis 2007; 22: 1389-93.

7. Madigan MR, Morson BC. Solitary ulcer of the rectum. Gut 1969; 10: 871-81.

8. Sharara Al, Azar C, Amr SS, et al. Solitary rectal ulcer syndrome: endoscopic spectrum and review of the literature. Gastrointest Endosc 2005; 62: 755-62.

9. Morio O, Meurette G, Desfourneaux V, et al. Anorectal physiology in solitary ulcer syndrome: a case-matched series. Dis Colon Rectum 2005; 48: 1917-22.

10. Samalavičius NE, Kildušis E. Hand-assisted laparoscopic suture rectopexy for complete rectal prolapse complicated by a solitary ulcer and obstructed defecation: a case report and review of the literature. J Med Case Rep 2013; 7: 133.

11. Brown S. The evidence base for rectal prolapse surgery: is resection rectopexy worth the risk? Tech Coloproctol 2014; 18 : 221-2.

12. Laubert T, Kleemann M, Roblick UJ, et al. Obstructive defecation syndrome: 19 years of experience with laparoscopic resection rectopexy. Tech Coloproctol 2013; 17: 307-14.

13. Tsuchida K, Okayama N, Miyata M, et al. Solitary rectal ulcer syndrome accompanied by submucosal invasive carcinoma. Am J Gastroenterol 1998; 93: 2235-8.

14. Samalavicius N, Ambrazevicius M, Kilius A, Petrulis K. Transanal endoscopic microsurgery for early rectal cancer: single center experience. Videosurgery Miniinv 2014; 9: 603-7.

15. Wałęga P, Kenig J, Richter P. Transanal endoscopic microsurgery combined with endoscopic posterior mesorectum resection in the treatment of patients with $\mathrm{T} 1$ rectal cancer - 3-year results. Videosurgery Miniinv 2014; 9: 40-5.

Received: 28.01.2015, accepted: 30.03.2015. 